\title{
Progress In SOFT X-RAY Detection: THE CASE OF EXOTIC HYDROGEN
}

\author{
J.-P. Egger, D. Chatellard and E. Jeannet
}

\section{Abstract}

Modern X-ray detection techniques with CCDs and their implications in the field of exotic hydrogen are discussed. Examples of recent achievements are presented and possible future developments are mentioned.

\section{Introduction}

An X-ray detector must have the best possible spatial and energy resolution, high quantum efficiency over a large energy region and a low particle background rate. Its use in both, the dispersive (position) mode in combination with a Bragg crystal spectrometer or in energy (non-dispersive) mode must be possible. In dispersive mode the position resolution is most important and the energy resolution is only used as a cut for background suppression. However, the overall efficiency (including solid angle, crystal reflectivity, etc.) is low. In nondispersive mode the energy resolution and the background rejection capabilities become most important.

Detectors for low energy $\mathrm{X}$-rays include gas filled proportional and scintillation counters, solid state devices such as conventional $\mathrm{Si}(\mathrm{Li})$ and $\mathrm{Ge}$ diodes, microchannel plates, silicon drift detectors and CCDs (charge-coupled devices). A detailed comparison of these detectors is presented in ref. [1] for the energy range from $1 \mathrm{keV}$ to $10 \mathrm{keV}$. As can be seen, CCDs have the best combined position and energy resolution except for new developments like microcalorimeters [2] or superconducting tunnel junctions [3] which both still are severely size-limited.

The CCD is essentially a silicon integrated circuit of the MOS type. The device consists of an oxide covered silicon substrate with an array of closely spaced electrodes on top. Each electrode is equivalent to the gate of a MOS transistor. Signal information is carried in the form of electrons. The charge is localized beneath the electrodes with the highest applied potentials because the positive potential of an electrode causes the underlying silicon to be depleted to a certain depth and thus have a positive potential which attracts the electrons. It is therefore common to say that the electrons are being stored in a "potential well".

"Charge coupling" is a technique to transfer a signal charge from under one electrode to the next (fig. 1). This is achieved by taking the voltage of the second electrode also to a high level, then reducing the voltage of the first electrode. Therefore, by sequentially pulsing the voltages on the electrodes between high and low levels, charges can be made to pass down an array of many electrodes with hardly any loss and very little noise.

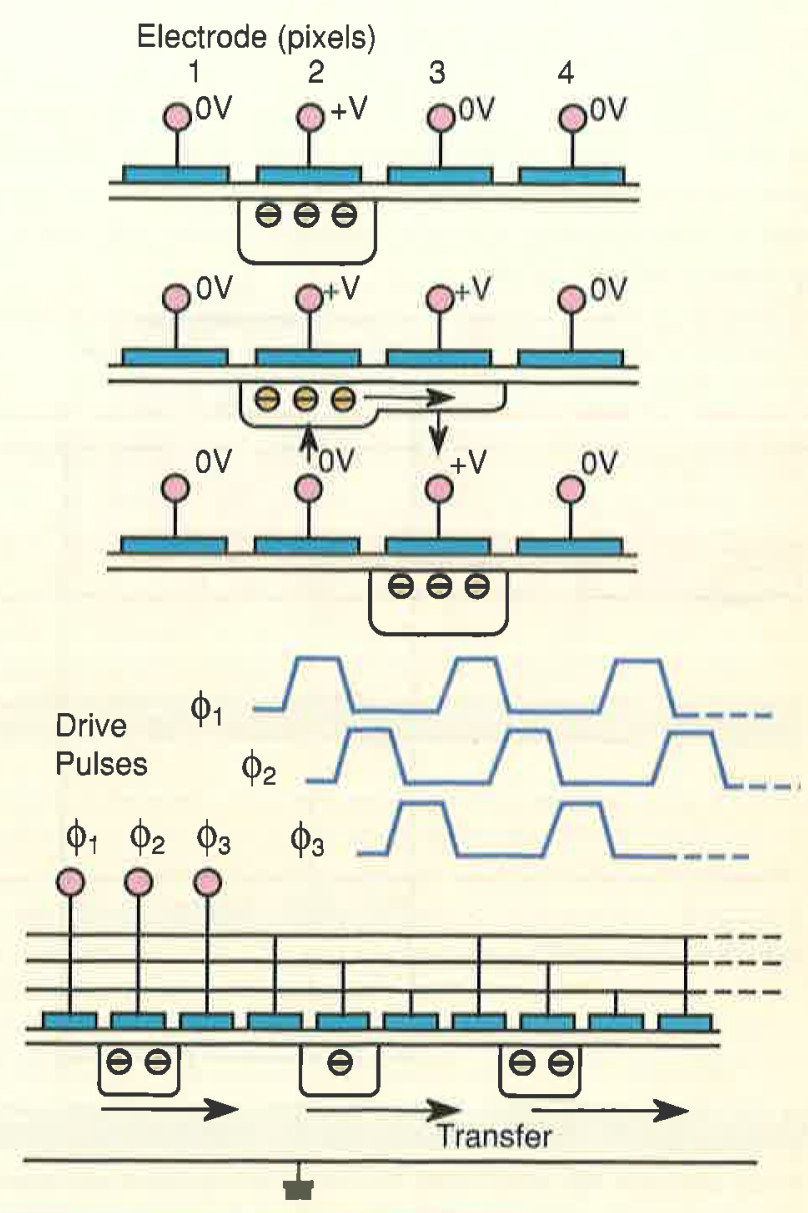

\section{FIGURE 1}

Charge signal transfer from one pixel to the next. One pixel contains three electrodes. The charges are in electrode No. 2 which has a voltage $+\mathrm{V}$ of $10 \mathrm{~V}$. The voltage of electrode No. 3 is set to the same level as that of No. 2. Simultaneously the voltage of No. 2 is reduced and the charges move to No, 3. A sequential pulsing of electrodes between two levels therefore allows a charge signal transfer over many electrodes (pixels). 
In sect. 2 we review the operation and performance of CCDs as X-ray detectors and present some energy and position spectra as examples. In sect. 3 some of the present and future physics in the field of exotic hydrogen, directly benefitting from improved $\mathrm{X}$-ray detection, is discussed. Finally, sect. 4 presents some general conclusions and an outlook including applications beyond basic nuclear and particle physics.

\section{Operation and performance of CCDs as X-ray detectors}

The CCDs [4] are operated in vacuum and cooled down to approximately $160 \mathrm{~K}$ in order to limit dark current and therefore allow for up to several hours of exposure time. They operate in a similar way to conventional silicon solid state detectors in that the incoming X-rays, following absorption by photoelectric effect, are converted to electron-hole pairs where edch pair requires $3.68 \mathrm{eV}$ for its creation. In contrast to the visible photon case the number of electrons created depends on the X-ray energy and a good energy resolution can therefore be achieved. A schematic view of the detection of visible photons, $\mathrm{X}$-rays and charged particles is given in fig. 2 .

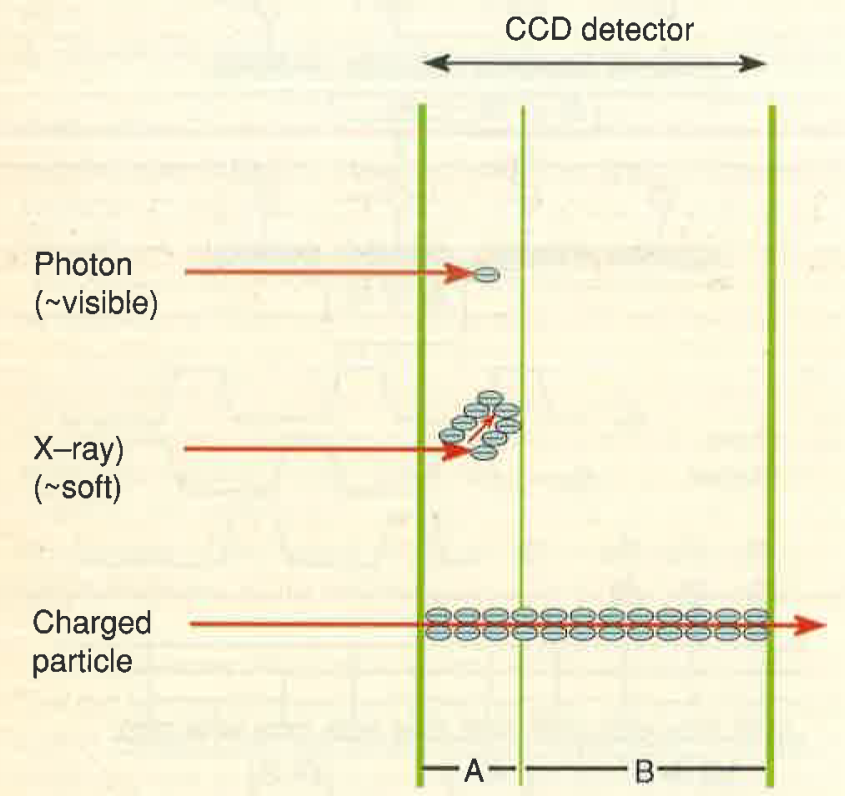

\section{FITURE 2}

Photon and particle detection in a CCD. The number of electron-hole pairs created is one per photon for visible light, about 270 per keV for $\mathrm{X}$-rays and approximately 80 per micron of Si for minimum ionizing charged particles. A corresponds to the depleted zone and $\mathbf{B}$ to the substrate.

The energy resolution of a CCD is given by where $\mathrm{N}$ is the r.m.s. transfer and readout noise of the $\mathrm{CCD}, \mathrm{F}$ the Fano factor and $\mathrm{E}$ the $\mathrm{X}$-ray energy. The conversion energy for an electron-hole pair in $\mathrm{Si}$ is $3.68 \mathrm{eV} .2 .355$ is simply the r.m.s.FWHM conversion factor. Lowest possible readout noise can be obtained by reducing output transistor noise and by using the "correlated double sampling" technique to eliminate reset noise [5]. However this requires slow-scan operation (reading time of one pixel is at least of the order of $50 \mu \mathrm{s}$ ) and therefore readout is slow. From the formula above, the best possible energy resolution with Si CCDs can be estimated by considering $\mathrm{N}^{2}$ very small. The result is $70 \mathrm{eV}$ FWHM at $2 \mathrm{keV}$ and $140 \mathrm{eV}$ FWHM at $8 \mathrm{keV}$. A small $\mathrm{N}^{2}$ can be achieved by using the so-called "skipper technique" [6] where the charge in a pixel is measured non destructively many times and then averaged. However this will further increase the readout time which is already long.

Examples of muonic hydrogen X-ray energy spectra are presented in fig. 3 and antiprotonic hydrogen is shown in fig. 4. The energy resolution is $150 \mathrm{eV} \mathrm{FWHM}$ at $2 \mathrm{keV}$ in fig. 3 and $140 \mathrm{eV}$ FWHM at $1.73 \mathrm{keV}$ in fig. 4. Fig. 5 shows an energy spectrum of ${ }^{55} \mathrm{Mn} \mathrm{X}$-rays from a ${ }^{55} \mathrm{Fe}$ source at $5.90 \mathrm{keV}$ and $6.49 \mathrm{keV}$. The energy resolution is $175 \mathrm{eV}$ FWHM. Work is in progress to further improve these energy resolutions by identifying all possible noise sources and minimizing them.

The CCD position resolution is simply given by the pixel size; in our case $22 \mu \mathrm{m} \times 22 \mu \mathrm{m}$. Up to four CCDs with a surface of $4.3 \mathrm{~cm}^{2}$ each $(770 \times 1152$ pixels $)$ can be operated simultaneously. Figure 6 presents a pionic hydrogen X-ray position spectrum after Bragg reflection on a crystal (dispersive mode). The corresponding energy resolution is approximately $40 \mathrm{meV}$ per pixel.

The CCD background rejection capabilities are very powerful and work as follows: events are collected into the CCD for a finite time and then read out. This collection time must not be too long, to avoid a double hit on a single pixel. In most of the cases, the energy of a soft X-ray is deposited into one single pixel. Sometimes, however, the energy is split between two pixels $(\approx 15-35 \%$ of the cases, depending on energy and CCD depletion depth). Background events like charged particles, neutrons and gamma, on the other hand, are usually split into several pixels. There are three reasons for this difference. Firstly, charged background particles tend to produce a larger lateral spread of electron-hole pairs of which some can leak to neighboring pixels. Secondly, the background events enter the detector from all directions, therefore having a higher probability to hit several pixels. The third reason can be understood by examining fig. 2 . When a suitable voltage is applied on the substrate (B), the electrons of the created pairs in B will migrate and some of them will eventually reach neighboring pixels whereas the X-ray electrons, confined in region $\mathrm{A}$, deposit their charge in one pixel. It was then possible to adjust the voltages for maximizing one pixel events with an X-ray source and maximizing multiple pixel events with other sources. A good event is then characterized by 


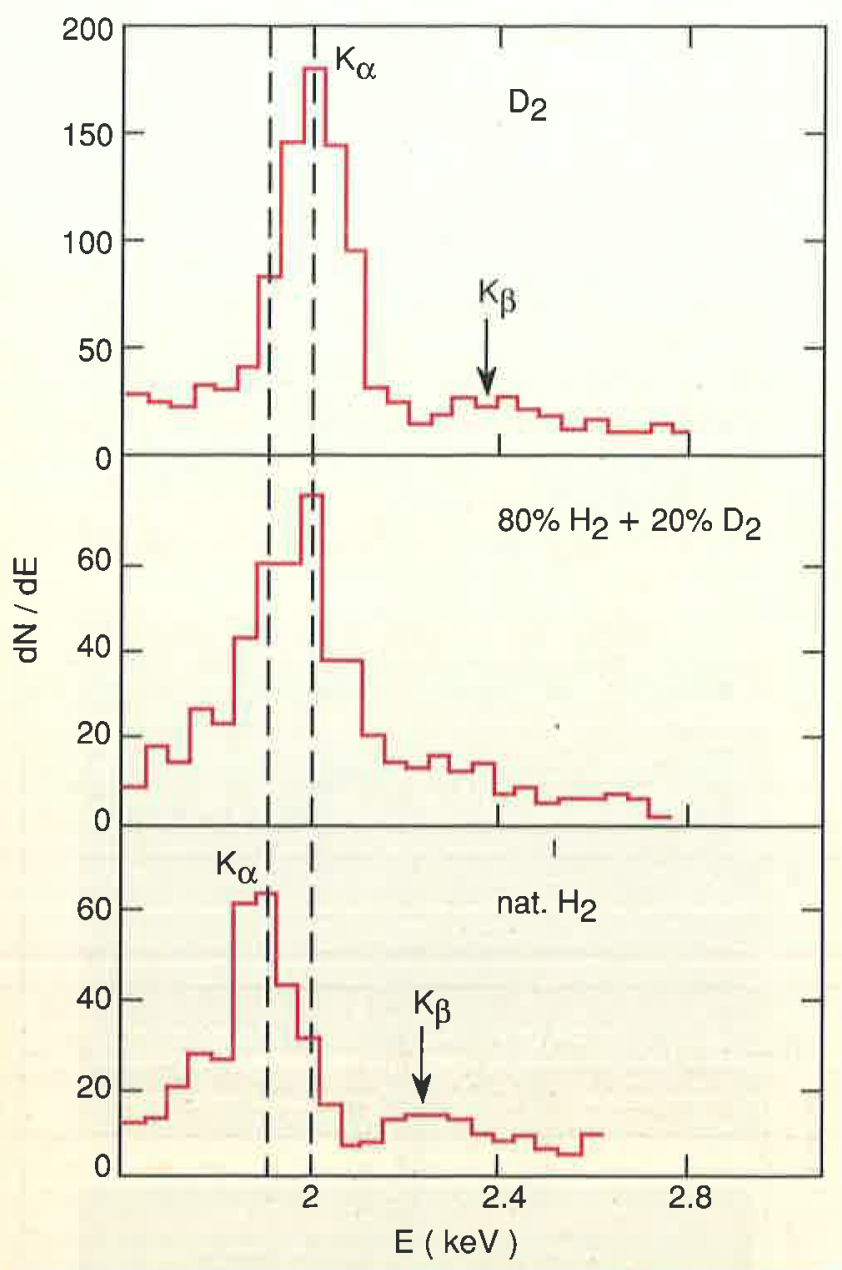

\section{FICIURE 3}

Raw muonic $\mathrm{X}$-ray spectra from natural $\mathrm{H}_{2}, \mathrm{HD}$ and $\mathrm{D}_{2}$ liquid targets detected by CCDs at a distance of $16 \mathrm{~cm}$ from the centre of the target. In addition to the target windows of $100 \mu \mathrm{m}$ Be a Kapton window of $12.5 \mu \mathrm{m}$ separated the target insulation vacuum from the $\mathrm{CCD}$ vacuum. No shielding was used. One channel corresponds to $46.6 \mathrm{eV}$. The energy resolution is approximately $150 \mathrm{eV}$ FWHM. The $\mathrm{K}_{\alpha}$ and $\mathrm{K}_{\beta}$ peaks for muonic hydrogen and deuterium are well separated (the $\mathrm{K}_{\alpha}$ positions at $1.90 \mathrm{keV}$ for $\mathrm{p} \mu$ and $2.00 \mathrm{keV}$ for $\mathrm{d} \mu$ are marked with dotted lines). $\mathrm{X}$-ray yields for higher $\mathbf{K}$ transitions are very small at liquid densities. Signal to background ratio is $\approx 10$. Even without a fit one can see directly by comparing the $\mathrm{K}_{\alpha}$ position in the 3 plots that there is a sizeable excited state $\mu$ transfer from $p \mu$ to $d \mu$ since the $\mathrm{HD} \mathrm{K}_{\alpha}$ line shape is already dominated by the $\mathrm{d} \mu$ contribution although there is only $20 \%$ deuterium present.

an isolated pixel with the proper energy surrounded by eight pixels which must be part of the noise peak. A simple lower limit on the surrounding pixels was used for figs 3 and 5. In figs 4 and

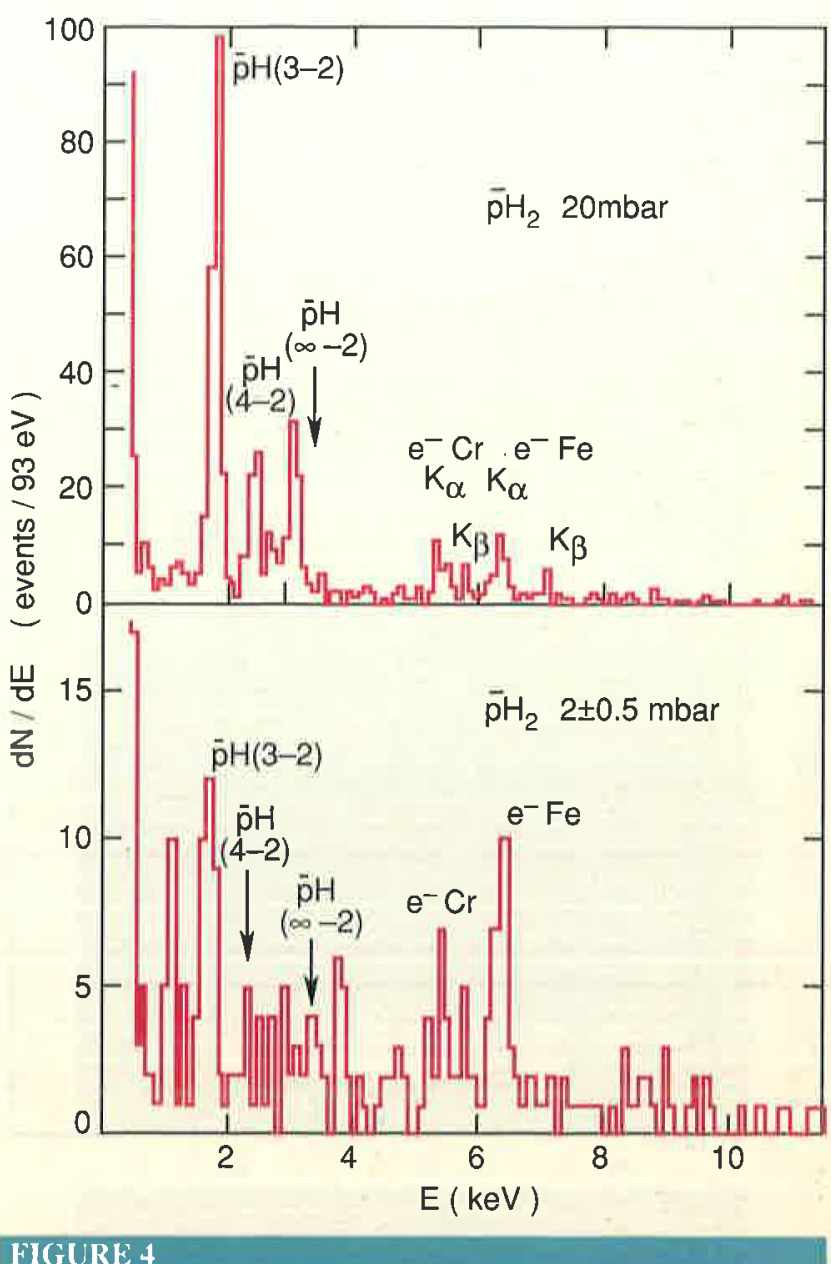

Antiprotonic hydrogen spectra taken with CCDs inside the cyclotron trap at LEAR for hydrogen target pressures of 2 and 20 mbar. This short measurement was carried out during the P118 test experiment for development of a research program on ultra low energy antiprotons at CERN. A $72 \mathrm{MeV} / c$ antiproton beam was used in slow extraction mode. One spill was necessary for each spectrum. The $\overline{\mathrm{p}} \mathrm{H}$ Balmer series $X$-rays are clearly visible. Signal/background at $20 \mathrm{mbar}$ is $\approx 20$. Fe and $\mathrm{Cr}$ electronic $\mathrm{X}$-rays are due to the stainless steel grid necessary to protect the CCDs from the active injection device (kicker) and pulsed extraction electrodes used in this test. A careful examination of the 2 mbar spectrum in the $8-10 \mathrm{keV}$ energy region shows that a clean measurement of the $\bar{p} H K_{\alpha}$ line could be carried out with a smalt internal bremsstrahlung contribution as the only background.

6 more sophisticated criteria were introduced [7]. This resulted in the loss of some events, but background was reduced to almost zero. Indeed in fig. 6 which is a position spectrum in the focal plane of a crystal spectrometer [8] an additional energy cut was performed and background was lowered to 1 event per $\mathrm{cm}^{2}$ and 
per 120 hours in the $\pi \mathrm{E} 3$ area at the Paul Scherrer Institute (PSI) with beam on. The background rejection capabilities are further illustrated in fig. 7. After irradiation of the CCDs the computergenerated images of CCD surfaces without background rejection are shown on the left and after rejection on the right.

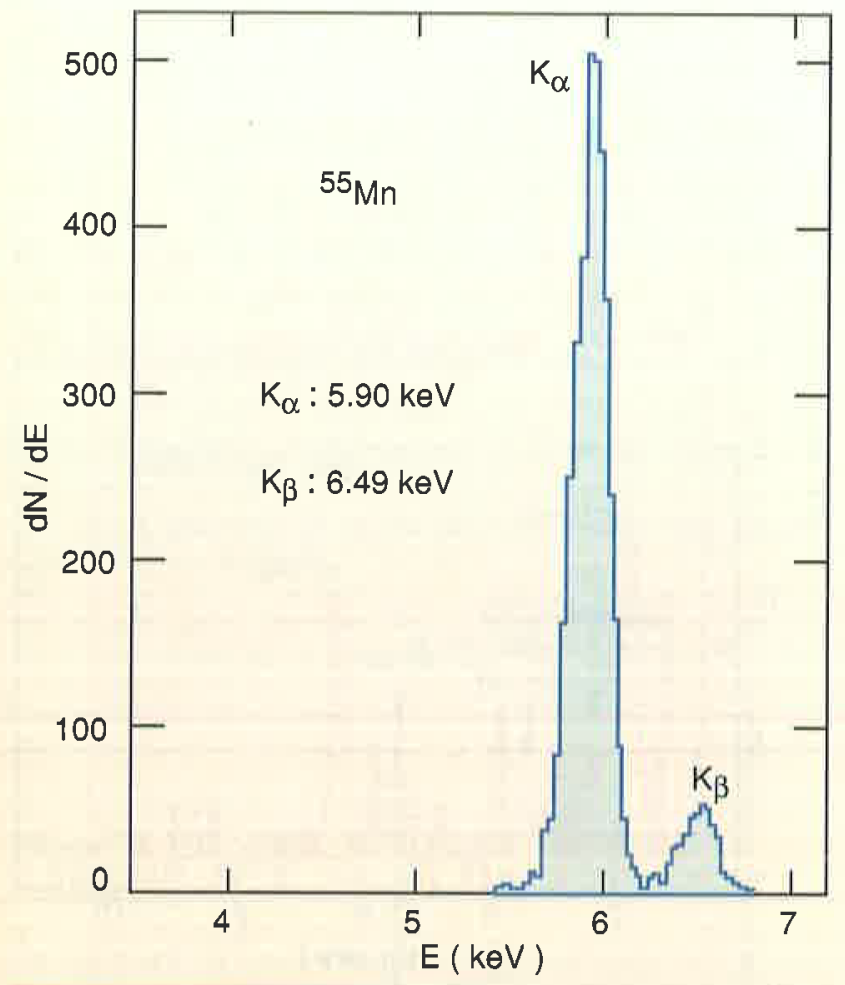

\section{FIGURE 5}

Energy spectrum of ${ }^{55} \mathrm{Mn}$ X-rays from a ${ }^{55} \mathrm{Fe}$ source at $5.90 \mathrm{keV}$ and $6.49 \mathrm{keV}$, The energy resolution is $175 \mathrm{eV}$ FWHM.

The theoretical CCD X-ray detection efficiency as a function of energy is given in fig. 8 [4]. However X-ray loss due to windows, target liquid or gas, etc. is not included. The sharp variation of the efficiency at $1.84 \mathrm{keV}$ corresponds to the Si absorption edge. The effect of this edge can be seen in the hydrogen spectrum in fig. 3 (asymmetry of the $\mathrm{K}_{\alpha}$ peak). A good way to measure the $\mathrm{CCD}$ efficiency including $\mathrm{X}$-ray losses is to detect $\mathrm{X}$ rays from antiprotonic nitrogen at low gas pressure where the circular transitions dominate with a yield of practically $100 \%$ [9]. An example (although with little statistics) is presented in fig. 9.

\section{Examples of exotic hydrogen physics}

Any particle with negative charge and sufficiently long life time can be captured in the Coulomb field of an atomic nucleus thus forming a hydrogen-like "exotic atom". The particle which initially may be trapped in a state with high principal quantum number $\mathrm{n}$ runs through its atomic cascade by emitting atomic $\mathrm{X}$ rays or electrons of the host atom (Auger effect). This cascade takes of the order of $10^{-15} \mathrm{~s}$ and the life time of the trapped particle must be long compared to this number. The most common of these negatively-charged particles are the muon, pion, kaon and antiproton. All these particles have masses which are large as compared to the electron mass. As the Bohr radius is inversely proportional to the mass, the spatial dimensions of exotic atoms are scaled down by the mass ratio of the trapped particle over the electron. In the case of a hydrogen atom these exotic systems are very simple and it is possible to study them at zero energy in a straightforward way by precise determination of the X-ray energies of the innermost transitions.

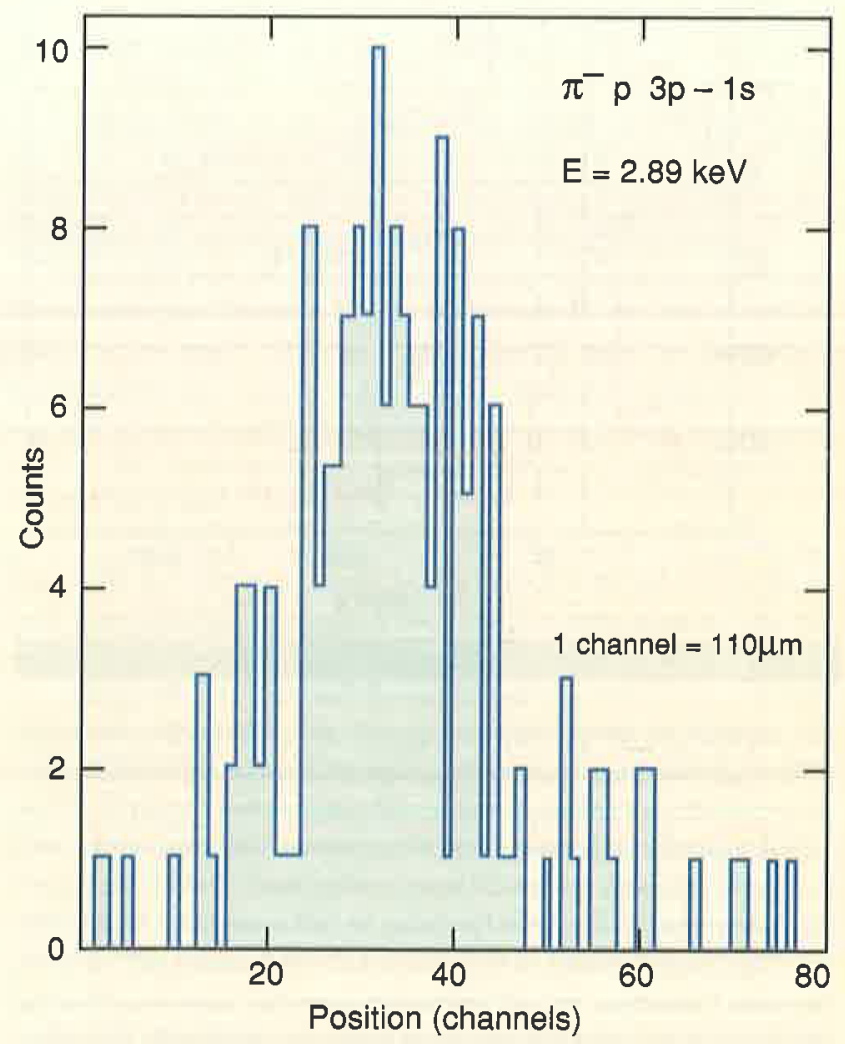

\section{FICURE 6}

Pionic hydrogen 3P-1S $\left(\mathrm{K}_{\beta}\right) \mathrm{X}$-ray position spectrum in the focal plane of a $\mathrm{Si}$ crystal spectrometer. One channel corresponds to 5 pixels $(110 \mu \mathrm{m})$. In this experimental set-up the energy resolution of one pixel was approximately $40 \mathrm{meV}$. The overall resolution of $\approx 3 \mathrm{eV}$ FWHM was therefore given by the crystals. Background suppression was carried out according to [7]. Signal/background ratio is $\approx 15$. The line shape was taken from a fit to the argon $\mathbf{K}_{\alpha 1}$ calibration spectrum. 

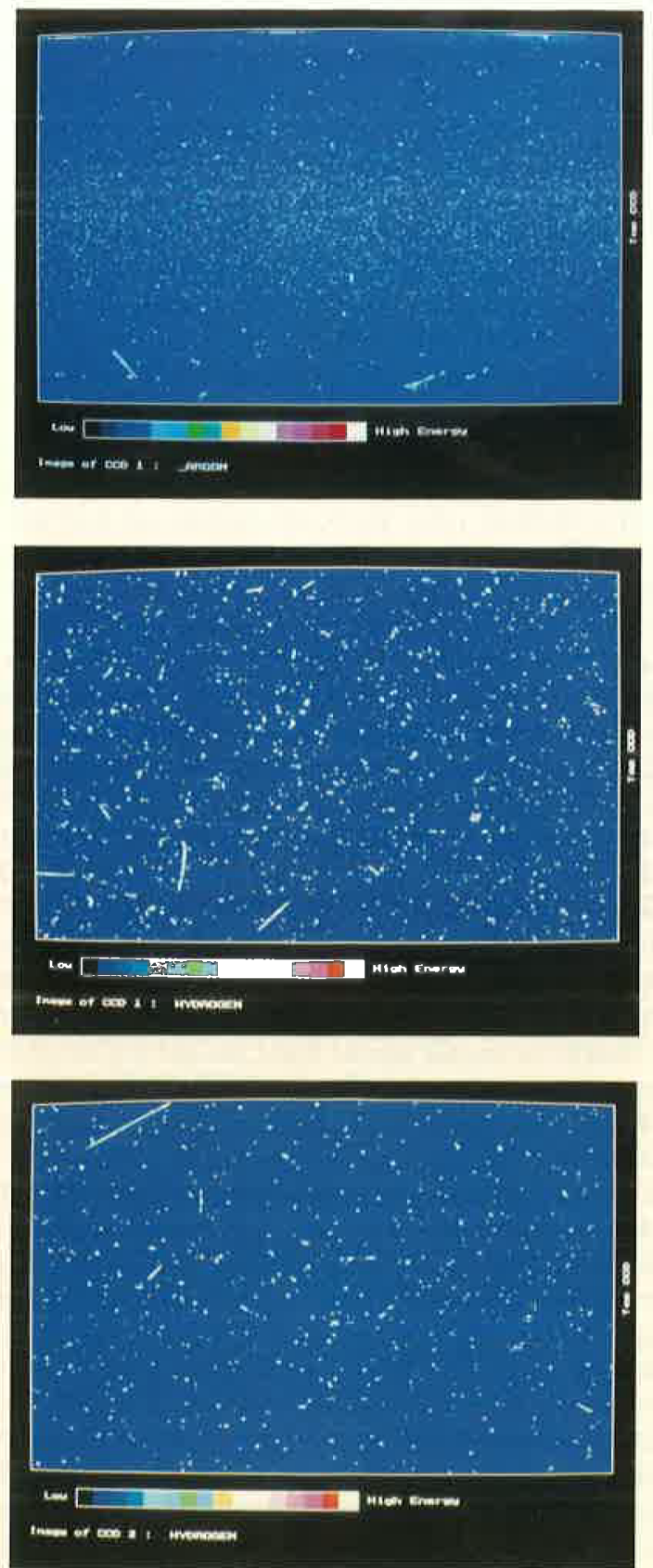

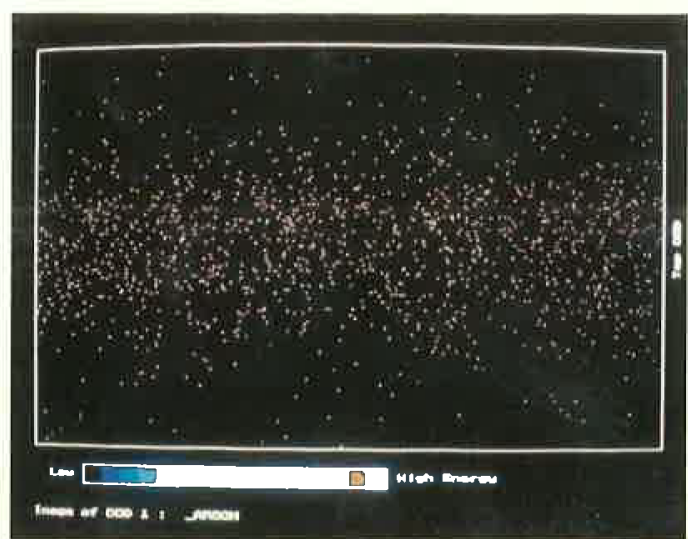

(a)

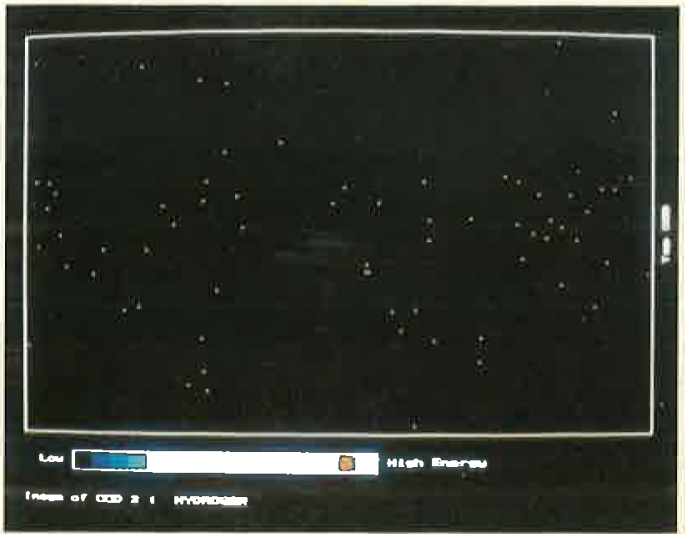

(b)

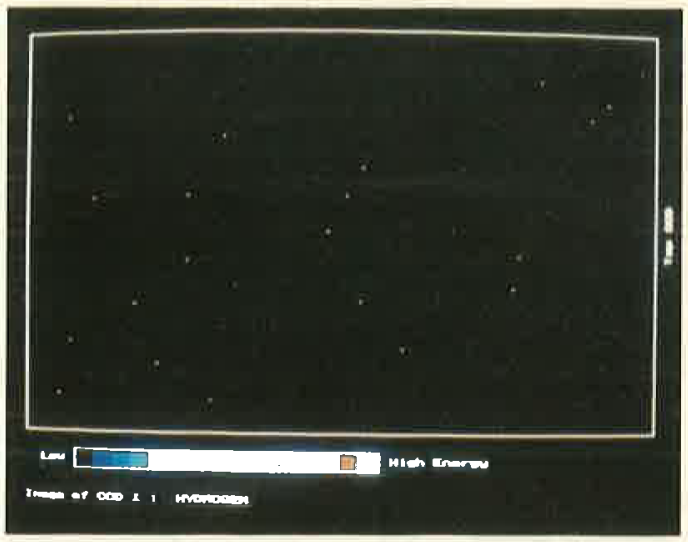

(c)

\section{FIGURE 7}

Computer-generated images of CCD surfaces irradiated with different particles. These figures present the background suppression capabilities of CCDs placed in the focal plane of a Si crystal spectrometer. The images on the left correspond to the raw spectra whereas the result of the background suppression is displayed on the right. The 3 cases are (a) a strong Ar $\mathrm{K}_{\alpha} \mathrm{X}$-ray calibration source (2.95 keV) obtained by fluorescence of ${ }^{55} \mathrm{Mn} \mathrm{X}$-rays in Ar gas plus little background, (b) some pionic hydrogen X-rays (3P-1S, $2.88 \mathrm{keV}$ ) and background and (c) a few pionic hydrogen X-rays in a severe background ( $\pi \mathrm{E} 3$ channel at PSI, $200 \mu \mathrm{A}$ primary proton current, slits wide open, $2.5 \mathrm{~m}$ from channel exit). 


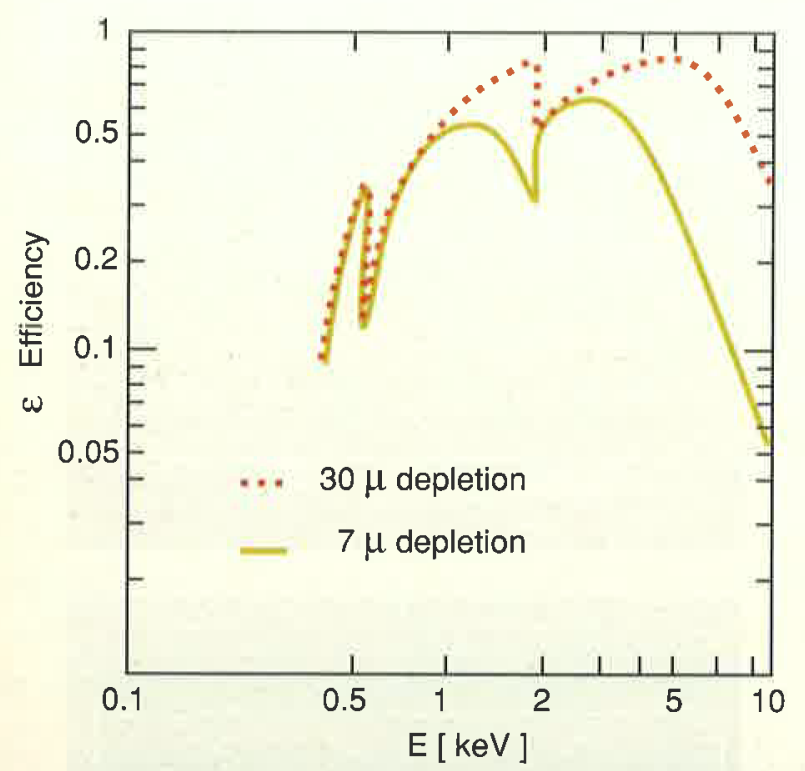

\section{FIGURE 8}

Efficiency as a function of X-ray energy for CCDs with $7 \mu \mathrm{m}$ and $30 \mu \mathrm{m}$ depletion layer.

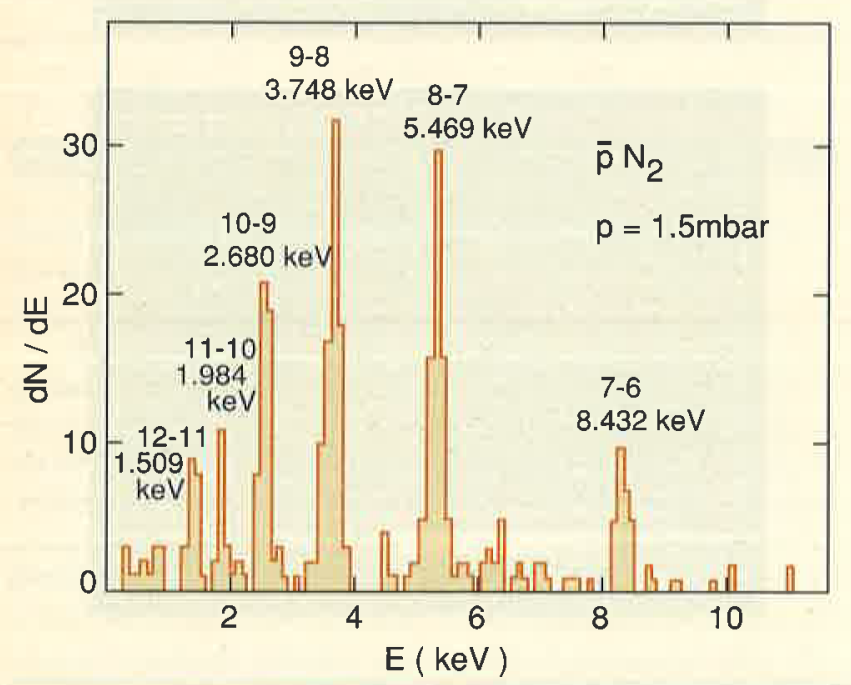

\section{FI(HURE 9}

CCD measurement of antiprotonic nitrogen X-rays for a target pressure of 1.5 mbar. At this low pressure the yield for circular transitions ( $\rightarrow n-1, I$ max.) is practically $100 \%$. The different peaks give therefore an experimental efficiency curve including $\mathrm{X}$-ray losses in windows, etc.

\subsection{Spectroscopy of muonic hydrogen}

The predictions of quantum mechanics can be verified to a very high degree of accuracy in muonic hydrogen in view of its simplicity and because some higher order corrections are not negligible like in ordinary hydrogen.

Let's take the 3D-3P transition as an example. At low hydrogen gas pressures where Stark mixing is strongly suppressed, the transition probability between levels with the same principal quantum number is very small. However this probability can be increased by shining infrared light with the corresponding frequency on the $\mu^{-}$p system to stimulate the transition. An increase in 3D-3P transitions will increase the $3 \mathrm{P}-1 \mathrm{~S}\left(\mathrm{~K}_{\beta}\right)$ versus the $2 \mathrm{P}-1 \mathrm{~S}\left(\mathrm{~K}_{\alpha}\right)$ yield. The frequency of the stimulated transition can then be deduced from the observation of the $\mathrm{K}_{\beta} / \mathrm{K}_{\alpha}$, intensity ratio. The $\mathrm{X}$-ray energies are $2.25 \mathrm{keV}$ and $1.90 \mathrm{keV}$. This type of experiment was proposed at PSI [10]. First tests should begin this year.

\subsection{Muon catalyzed fusion ( $\mu \mathrm{CF}$ )}

The $\mu \mathrm{CF}$ process is started by injecting a free muon into a mixture of hydrogen isotopes where it is stopped. Following the initial Coulomb capture, the muon cascades to lower levels via Stark mixing, Auger, inelastic and radiative processes. This muonic cascade, even in the simplest muonic atoms (muonic hydrogen $\mathrm{p} \mu$, deuterium $\mathrm{d} \mu$ and tritium $\mathrm{t} \mu$ ) is not sufficiently understood quantitatively.

In particular the probability $\mathrm{q}_{1 \mathrm{~s}}$ to reach the ground state of a given muonic hydrogen isotope has never been directly measured. A precise knowledge of $\mathrm{q}_{1 \mathrm{~s}}$ gives information about muon transfer in excited atomic states and defines therefore the initial conditions for the complex cycle of muon-induced processes. Figure 3 presents a first direct measurement of muonic $\mathrm{X}$-rays from natural $\mathrm{H}_{2}, \mathrm{HD}$ and $\mathrm{D}_{2}$ liquid targets measured with CCDs during a test run on the $\mu \mathrm{E} 4$ channel at PSI [11]. Even without a fit, the comparison of the $\mathrm{K}_{\alpha}$ position in the three plots shows a sizeable $\mu$ transfer from $\mathrm{p} \mu$ to $\mathrm{d} \mu$ since the $\mathrm{HD} \mathrm{K}_{\alpha}$ line shape is clearly dominated by the $d \mu$ contribution although there is only $20 \%$ deuterium present. Systematic measurements will start this year.

\subsection{Antiprotonic hydrogen}

Since the antiproton is a strongly interacting particle the energy of the 1S level in antiprotonic hydrogen will be shifted $(\varepsilon)$ and broadened $(\Gamma)$ due to the strong interaction. In order to determine these parameters, accurate energy measurements are needed. Furthermore, calculations by Borie and Leon [12] show that $\mathrm{K}$ and $\mathrm{L}$ X-ray intensities are very low except at the lowest hydrogen gas pressures. Therefore the maximizing of stopping density and the reduction of background is of prime importance. An interesting way of obtaining a high stopping density of antiprotons in a low density gas target is to use a "cyclotron trap" [13].The principle of the trap is to wind up the range curve of the 
incoming charged particle beam in a weakly focusing magnetic field of the order of $2.5 \mathrm{~T}$ produced by a superconducting split coil magnet. After radial injection the particles spiral towards the centre while loosing energy in a few thin degraders and finally the target gas. Obviously the trap works best for particles which are stable (antiprotons) or have a long life time (muons). Figure 4 presents antiprotonic hydrogen spectra taken with CCDs inside this cyclotron trap for hydrogen target pressures of 2 and 20 mbar [14]. The quality of the data obtained from this test at LEAR (CERN) shows that the strong interaction parameters $\varepsilon_{1 S}$ and $\Gamma_{\text {IS }}$ could be determined with significantly better accuracy. Therefore a precise comparison with the different theoretical predictions could be made. A detailed experimental and theoretical review of antiprotonic hydrogen up to 1989 by C.J. Batty can be found in ref. [15].

\subsection{Pionic hydrogen}

The measurement of the strong interaction shift $\varepsilon_{\mathrm{IS}}$ and broadening $\Gamma_{I S}$ is a suitable way to study the pion-nucleon strong interaction at zero energy since these quantities can be directly related to the $S$-wave scattering lengths $a_{1}$ (isospin $1 / 2$ ) and $a_{3}$ (isospin 3/2). The scattering lengths can also be obtained indirectly from dispersion relations using phase shifts from low energy elastic $\pi p$ scattering and charge exchange measurements and extrapolating to zero energy [16]. This procedure is indirect since extensive theoretical treatments of the experimental data are necessary. In addition, results from different scattering experiments lead to solutions for the low energy parameters which are mutually inconsistent [17]. This is not surprising since, because of their tendency to decay rapidly, low energy pions are extremely difficult objects to handle in an experiment.

In a first step at PSI $\varepsilon_{1 S}$ was measured with an accuracy of $\approx 5 \%$ [8]. The result is $\varepsilon_{1 \mathrm{~S}}=-7.12 \pm 0.32 \mathrm{eV}$ (attractive) yielding the scattering length combination $1 / 3\left(2 a_{1}+a_{3}\right)=0.086$ $\pm 0.004 \mathrm{~m}_{\pi}^{-1}$. Work is in progress to improve the set-up for an $\varepsilon_{1 \mathrm{~S}}$ measurement at the $1 \%$ and $\Gamma_{\text {IS }}$ at the $10 \%$ level. Run time for this experiment was scheduled on the new $\pi \mathrm{E} 5$ beam line last summer with the cyclotron trap, a new high resolution crystal spectrometer and new large size CCDs [18].

An important application of the $\pi \mathrm{N}$ scattering parameters at zero energy is the investigation of the low energy structure of QCD through the calculation of the so-called pion-nucleon sigma-term. A summary of the present situation may be found in [19].

\subsection{Kaonic and hyperonic hydrogen}

Early measurements of $\varepsilon_{1 S}$ and $\Gamma_{1 S}$ in kaonic hydrogen [20] were plagued by small statistics, large backgrounds and Stark effect since a liquid hydrogen target had to be used in order to have a sufficient stopping density. An improved experiment could be carried out at the future KAON (Vancouver) or at a $\Phi-$ factory like DAФNE (Frascati) with CCDs. However even with a precise measurement of $\varepsilon_{1 S}$ and $\Gamma_{1 S}$, some problems arise when the $\mathrm{s}$-wave scattering length is deduced. From the analysis of low energy $\mathrm{K}^{-} \mathrm{p}$ scattering, the $\mathrm{K}^{-} \mathrm{p}$ scattering length is known to be positive. However this is due to the presence of the baryonic resonance $\Lambda(1405)$ which strongly couples to the isospin zero channel of the $\mathrm{K}^{-}$p system [21].

The energy of the sigmaonic hydrogen $2 \mathrm{P}-1 \mathrm{~S}$ transition is of the order of $10 \mathrm{keV}$. This transition could be measured simultaneously with the kaonic hydrogen $2 \mathrm{P}-1 \mathrm{~S}$ transition since the $\mathrm{K}^{-}$can be stopped and the $\Sigma^{-}$produced and stopped in the same hydrogen target $\left(\mathrm{K}^{-} \mathrm{p} \rightarrow \Sigma^{-} \pi^{+}\right.$with a very low energy $\Sigma^{-}$ appears in $\approx 44 \%$ of the cases). For the $\Sigma^{-} p$ atom the $\Sigma(1385)$ resonance which is also below the $\mathrm{K}^{-} \mathrm{p}$ threshold should not, because of its isospin, complicate the situation in the way the $\Lambda(1405)$ does in the $\mathrm{K}^{-}$p system.

$\mathrm{Xionic}$ and omegaonic hydrogen $\mathrm{X}$-rays might also be obtained at KAON or other places (COSY, Jülich, for example) with a set-up similar to the so-called "Gatchina configuration" [22].

\section{Conclusions and outlook}

CCDs have a unique potential as $\mathrm{X}$-ray detectors for energies between $1 \mathrm{keV}$ and $10 \mathrm{keV}$ since no other $\mathrm{X}$-ray detector can match their overall performance at this time. However, further significant improvements in detector quality will be hard to achieve without major programs of development. As examples, let us mention a few areas where such programs might prove useful:

(a) In order to overcome the natural limit for the energy resolution of $70-140 \mathrm{eV} \mathrm{FWHM,} \mathrm{the} \mathrm{new} \mathrm{developments} \mathrm{on}$ microcalorimeters and superconducting tunnel junctions are very promising but the serious problem of size limitation has to be solved before these detectors are of practical use.

(b) A more constant detector efficiency as a function of soft Xray energy can be obtained with Ge CCDs (the Ge K absorption edge is at $11.1 \mathrm{keV}$ versus $1.84 \mathrm{keV}$ for $\mathrm{Si}$ ). In addition $\mathrm{Ge}$ is a better semiconductor for $\mathrm{X}$-ray detection above $10 \mathrm{keV}$ because of its higher $\mathrm{Z}$ value. However the $\mathrm{Ge}$ oxides needed for the CCD surface and depletion layer do not exist in forms comparable to Si.

(c) An increase of the CCD efficiency below $1.3 \mathrm{keV}$ (fig. 8) can be obtained by decreasing the thickness of the polysilicon electrodes and oxide layers at the CCD surface in order to reduce $\mathrm{X}$-ray absorption [23]. Back illumination after etching off the substrate is also a possibility but might interfere with charged particle rejection.

(d) CCD readout is slow if excellent pixel transfer efficiency is needed, as is in the case of good energy resolution. CCD readout could be speeded-up by incorporating an array instead of a single on-chip charge detection amplifier plus output electronics. 
Tests have shown that CCDs can work in strong magnetic fields and directly in the isolation vacuum of a cold target or in a accelerator or beam-line vacuum.

Many other applications for X-ray CCDs can be imagined. They include:

(i) X-ray astronomy. The bulk of the detectable X-ray flux from outside the solar system and the emission and absorption lines of the most abundant elements lie in the energy region from 0.1 to $10 \mathrm{keV}$, nicely covering the $\mathrm{CCD}$ detection range. Problems arise in space with cooling and irradiation damage. Tests at PSI and LEAR by exposing CCDs to different particle beams could help to understand the irradiation damage problem. Future X-ray space programs include the Russian mission SPECTRUM X-G and the European effort XMM.

(ii) Detection of single low energy electrons and positrons. Single electrons and positrons in the energy range up to $10 \mathrm{keV}$ should interact in a CCD like X-rays of the same energy, provided the surface layer of the CCDs (electrodes etc.) is $<1$ $\mu \mathrm{m}$. In experiments like the muonium to antimuonium conversion at PSI, a unique identification of positrons should be possible. However CCD readout speed and triggering have to be improved.

(iii)Biology and solid state physics. With the advent of the new synchrotron light sources at high intensity storage rings like ESRF at Grenoble, many new experiments requiring high position and energy resolution for X-ray detection become feasible. These include protein crystallography and autoradiography in biology [24], soft X-ray spectroscopy and materials research. CCDs are promising detectors in this field, especially if faster readouts can be built.

It should be noted however that CCDs of current design cannot be used in the energy region above $15 \mathrm{keV}$ which includes most medical X-rays for fundamental reasons ( $\mathrm{Si}$ becomes transparent to these X-rays). A new development of CCDs based on GeAs technology would extend the energy region up to approximately $100 \mathrm{keV}$. Plans for such a development exist [25].

\section{Acknowledgements}

We would like to thank all our colleagues that collaborated with us, especially our friends at IMP-ETHZ, PSI and Vienna. This work was partially funded by the Swiss National Science Foundation.

\section{References}

[1] J.L. Culhane, Nucl. Inst. and Meth. A310 (1991) 1.

[2] D. McCammon et al., Proc. Low Temp. Detectors for Neutrinos and Dark Matter, 1990, Gif-sur-Yvette (in press).

[3] D. Twerenbold, Physica C168 (1990) 381 (and references therein).
[4] EEV (English Electric Valve), Waterhouse Lane, Chelmsford, Essex, CM1 2QU, England.

[5] D. Varidel, J.-P. Bourquin, D. Bovet, G. Fiorucci and D. Schenker, Nucl. Inst. and Meth. A292 (1990) 147.

[6] J. Janesick et al., SPIE - Proceedings, VoI. 1242, Symposium on Electronic Imaging, Santa Clara, CA, USA (1990).

[7] D. Sigg, Diplomarbeit, ETHZ-IMP (1990).

[8] W. Beer, M. Bogdan, P.F.A. Goudsmit, H.J. Leisi, A.J. Rusi El Hassani, D. Sigg, St. Thomann, W. Volken, D. Bovet, E. Bovet, D. Chatellard, J.-P. Egger, G. Fiorucci, K. Gabathuler and L.M. Simons, Phys. Lett. B261 (1991) 16.

[9] R. Bacher, P. Blüm, D. Gotta, K. Heitlinger, M. Schneider, J. Missimer, L.M. Simons and K. Elsener, Phys. Rev. A38 (1988) 4395.

[10] F. Kottmann, C. Petitjean, L.M. Simons, D. Taqqu, D. Chatellard, M. Denoréaz, J.-P. Egger, E. Jeannet, F. Ciocci, G. Dattoli, A. Doria, G.P. Gallerano, L. Giannessi, G. Giubileo, G. Messina, A. Renieri, A. Vignati, F. Della Valle, E. Milotti, C. Rizzo, A. Vacchi and E. Zavattini, PSI proposal R-92-06.1.

[11] P. Ackerbauer, P. Baumann, E.D. Bovet, W.H. Breunlich, T. Case, D. Chatellard, K.M. Crowe, H. Daniel, J.-P. Egger, F.J. Hartmann, E. Jeannet, M. Jeitler, P. Kammel, B. Lauss, K. Lou, V. Markushin, J. Marton, C. Petitjean, W. Prymas, W. Schott, R.H. Sherman, T. von Egidy, P. Wojciechovski and J. Zmeskal, PSI proposal R-81-05 and PSI Nuclear and Particle Physics Newsletter (1991) 53 .

[12] E. Borie and M. Leon, Phys. Rev. A21 (1980) 1460.

[13] L.M. Simons, Phys. Scripta T22 (1988) 90.

[14] E.C. Aschenauer, D. Cauz, P. DeCecco, J. Eades, K. Elsener, G. Gorini, D. Horvath, I. Krafcsik, V. Lagomarsino, G. Manusio, R. Poggiani, L.M. Simons, G. Testera, G. Torelli and F. Waldner, The P118T test experiment at LEAR (CERN) .

[15] C.J. Batty, Rep. Prog. Phys. 52 (1989) 1165.

[16] O. Dumbrajs et al., Nucl. Phys. B216 (1983) 277 (and references therein).

[17] J. Gasser, E. Leutwyler and M.E. Sainio, Phys. Lett. B253 (1991) 252 (and references therein).

[18] E.C. Aschenauer, A. Badertscher, W. Beer, D. Chatellard, M. Denoréaz, J.-P. Egger, K. Gabathuler, P.F.A. Goudsmit, E. Jeannet, H.J. Leisi, E. Matsinos, J. Missimer, H.Ch. Schröder, D. Sigg, L.M. Simons and Z.G. Zhao, PSI Nuclear ant Particle Physics Newsletter (1991) 55.

[19] J. Gasser, H. Leutwyler, M.P. Locher and M.E. Sainio, Phys. Lett. B213 (1988) 85 and ref. [17].

[20] J.D. Davies et al., Phys. Lett. B83 (1979) 55; M. Izycki et al., Z. Physik A297 (1980) 11; P.M. Bird et al., Nucl. Phys. A404 (1983) 482. 
[21] F. Scheck, Leptons, Hadrons and Nuclei, North-Holland, Amsterdam (1983) 79.

[22] V.I. Marushenko et al., JETP Lett. 23 (1976) 72.

[23] C. Castelli, A. Wells, K. McCarthy and A. Holland, Nucl. Inst. and Meth. A310 (1991) 240.

[24] A.R. Faruqi, Nucl. Inst. and Meth. A310 (1991) 14.

[25] P. Seitz, PSI-Zurich (private communication).

\section{Address:}

J.-P. Egger, D. Chatellard and E. Jeannet

Institut de Physique de l'Université

Breguet 1

CH-2000 Neuchâtel (Switzerland)

Received on July 1992. 